\title{
The effective estimation of skid resistance of SMA pavements in relevant aspect of safety and durability of asphalt roads
}

\author{
Beata Stankiewicz ${ }^{1, *}$, Pawel Slabonski ${ }^{2}$, and Emilia Slabonska ${ }^{2}$ \\ ${ }^{1}$ Faculty of Civil Engineering, Opole University of Technology, Poland \\ ${ }^{2}$ Research Engineering Institute Labor Aquila, Poland
}

\begin{abstract}
The role of skid resistance of road pavements in ensuring safety of road traffic becomes particularly important when such surfaces become moist or wet. The various types of aggregates and special additives play an important role in asphalt mixtures like SMA, because they fill voids in paving mix and improve the cohesion of asphalt binder. The paper presents the evaluation of skid resistance of five kinds of SMA pavements, during exploitation, based on macro-texture and micro-texture comprehensive analysis. Tests were performed on five research pavement sections made from SMA on roads located in the southern part of Poland. SRT 3 equipment and methodology were used to all estimation of pavement roughness. The micro-texture and macro-texture of asphalt surface in pavements evolve under the influence of traffic and synergistic environmental interaction. The experimental parts of SMA pavements were observed in relevant aspect of skid resistance and durability using electronic microscopy type KEYENCE VHX-6000 Digital. The results of evaluation were compared with data from a literature review.
\end{abstract}

\section{Introduction}

The role of skid resistance of road pavements in ensuring safety of road traffic becomes particularly important when such surfaces become moist or wet. Skid resistance is defined as a frictional resistance at the interface between a vehicle tire and the road surface. The measurement calculation of skid resistance is the friction coefficient, closely related to the surface texture, i.e. geometric description of its irregularities. The surface texture ensures draining water from the tire-pavement interface area. Deviations from a flat plane having wavelength between 0.5 and $50 \mathrm{~mm}$ are referred to as macro-texture and its characteristics defined by the upper layers technology and by the particle size distribution of the aggregate mix used in its production. Wave length below $0.5 \mathrm{~mm}$ are referred to as micro-texture which is defined by the resistance to polishing of coarse aggregate and the content of particles smaller than $2 \mathrm{~mm}$ in the aggregate mix used for the wearing course. Macro-

\footnotetext{
* Corresponding author: b.stankiewicz@po.opole.pl
} 
texture parameters include Mean Texture Depth $(M T D)$ determined by the volumetric method [1].

Pavement friction and texture characteristics are important aspects of road safety. Despite extensive studies conducted in the past decades, knowledge gaps still remain in good understanding of the relationship between pavement macrotexture and surface skid resistance [2]. Wet pavement skidding due to inadequate surface texture or friction contributes to $20 \%$ to $35 \%$ of all wet weather crashes. Many of the past studies of pavement surface texture and friction have deficiency in selecting the model forms or variables and interpreting the models and (or) the findings or have recommended further investigation [3]. Micro-texture depends on polishing resistance of coarse aggregate and content of fine aggregate in asphalt mixture. In actual traffic conditions the phenomenon of polishing of the protruding aggregate grains is caused by traffic. Due to the fact that micro-texture exists on a microscopic scale, it is very difficult to assess it [4]. The paper presents the evaluation of the skid resistance properties of five bituminous wear courses from the SMA grit mastic during exploitation, based on macro analysis and micro-texture analysis of the test specimens. Comprehensive research tests were carried out on the basis of five sections of the SMA pavement, located in the southern part of Poland.

\section{Mean Texture Depth for five various kinds of SMA pavements}

The European Standard EN 13036-1 Road and airfield surface characteristics - Test methods - Part 1: Measurement of pavement surface macrotexture depth using a volumetric patch technique specifies a method for determining the average depth of pavement surface macrotexture by careful application of a known volume of material on the surface and subsequent measurement of the total area covered. The technique is designed to provide an average depth value of only the pavement macrotexture and is considered insensitive to pavement microtexture characteristics. The test method is suitable for field tests to determine the average macrotexture depth of a pavement surface. When used in conjunction with other physical tests, the macrotexture depth values derived from the test method can be used to determine the pavement skid resistance capability, noise characteristics and the suitability of paving materials or finishing techniques. Mean Texture Depth was analyzed for five various types of SMA pavements (Fig. 1 - Fig. 3) from real location in the southern region of Poland.

\subsection{The sample No. 1}

The sample 1 (in Fig. 1) has been acquired from SMA 11, six years old pavement constructed with special innovative cement and extraordinary technology of implementation on a tested road part, for KR5-KR7 traffic category.

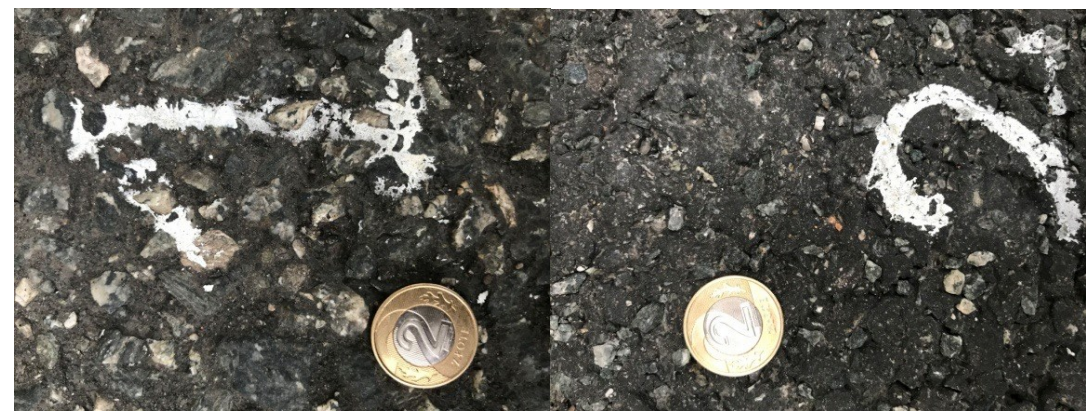

Fig. 1. The macro-textures of sample 1 from SMA 11 and sample 2 from SMA 11 PMB 45/80-45. 
The main core of the first sample No. 1 was $\varnothing 200 \mathrm{~mm}$ with $40 \mathrm{~mm}$ of thickness and the volume for granulated material $26,1 \mathrm{~mm}^{3}$. Mean Texture Depth (MTD) according to volumetric method achieved $1,1 \mathrm{~mm}$.

\subsection{The sample No. 2}

The second sample from SMA 11 PMB 45/80-45 (in Fig. 2) with different surface of macro-texture has been analysed by volumetric test using the same standard of parameters for sample - Ø $200 \mathrm{~mm}$ with $40 \mathrm{~mm}$ of thickness and the volume for granulated material $26,1 \mathrm{~mm}^{3}$. Mean Texture Depth aimed 0,86 mm. Standard type of SMA 11 PMB 45/80-45 pavement was constructed one year ago in a region near Czestochowa city for KR3-KR4 traffic road category. Results for both sample 1 and sample 2 were proper and higher than normative minimum $\mathrm{MTD}=0,6 \mathrm{~mm}$.

\subsection{The sample No. 3}

The next test has pertained to sample 3 from DENSIFALT SMA 11 with special high-value cement created for KR3-KR4, seven years ago. The main core of the sample No. 3 was $\varnothing$ $200 \mathrm{~mm}$ with $40 \mathrm{~mm}$ of thickness and the volume for granulated material $13,1 \mathrm{~mm}^{3}$. Mean Texture Depth (MTD) according to volumetric method achieved only 0,4 mm. Figure 2 explicates the macro-texture of sample 3, acquired from DENSIFALT SMA 11 from real, existing pavement.

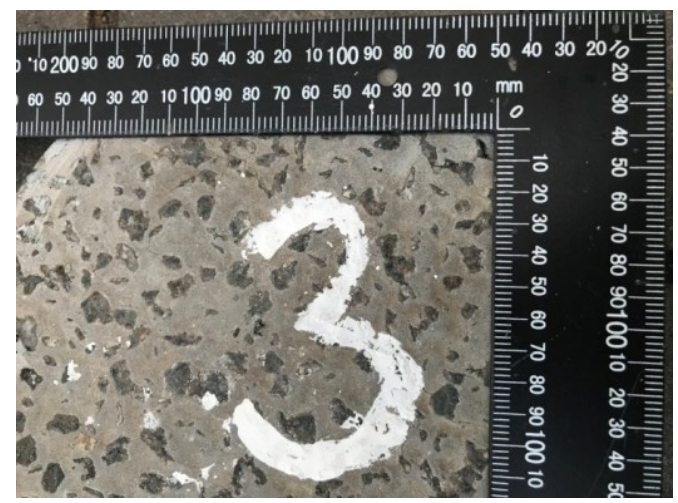

Fig. 2. The macro-texture of sample 3 from DENSIFALT SMA 11.

\subsection{The sample No. 4}

The next tested pavement (Fig. 3) was erected one year ago by SMA 11 45-80/80 HIMA with asphalt after high modification, suitable for traffic category from KR3 to KR7. The results of volumetric tests showed proper value of $\mathrm{MTD}=1,4 \mathrm{~mm}$ for analysed sample by main core of sample $\varnothing 200 \mathrm{~mm}, 40 \mathrm{~mm}$ of sample thickness and the volume for granulated material $26,1 \mathrm{~mm}^{3}$.

\subsection{The sample No. 5}

The sample number 5 (in Fig. 3.) was acquired from SMA 11 with asphalt type 50/70 rubber-modified. The objective pavement was finished one year ago for traffic category from KR3 to KR7. Main core of the sample $\varnothing 200 \mathrm{~mm}, 40 \mathrm{~mm}$ of sample thickness and the 
volume for granulated material $26,1 \mathrm{~mm}^{3}$ were compatible with different samples. Mean Texture Depth reached 1,4 $\mathrm{mm}$ for the fifth test sample.

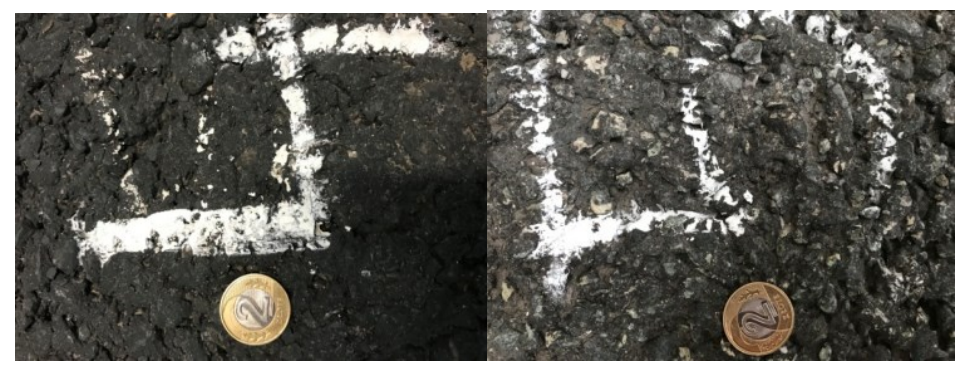

Fig. 3. The sample No. 4 and test sample No. 5 with specific, individual macro-texture.

\section{Reliable Skid Resistance for objective pavements}

Measurement of skid resistance is restricted by the need to measure the forces acting on a rubber wheel or slider while it is dragged across the surface. The skid resistance of a road is affected by its surface texture, and in particular, by texture with wavelengths less than 10 $\mathrm{mm}$. There are four classes of pavement state according to Skid Resistance Tester STR 3 by Barum Bravura test for $1 \mathrm{~km}$ part of a road:

- A standard - good technical standard - Reliable Skid Resistance $\geq 0,52$,

- B - satisfactory state - Reliable Skid Resistance $0,37 \div 0,51$,

- $\quad$ C - non satisfactory state - Reliable Skid Resistance $0,30 \div 0,36$,

- $\quad$ D - poor technical standard - Reliable Skid Resistance $\leq 0,29$ [5].

Skid Resistance Tester STR 3 with Barum Bravura test for $1 \mathrm{~km}$ part of road in a third location, for pavement created by DENSIFALT SMA 11 with special high-value cement designed for KR3-KR4, which had erected seven years ago, achieved non satisfactory level of C class of complex classification with 0,30 (Reliable Skid Resistance). The roughening intervention is needed for the objective pavement.

\section{Microscope scale analysis of surface for test samples}

Definitions of various scales of texture such as macrotexture and microtexture, the need for pavement microtexture and methods used to measure pavement texture. It has been well established that the surface texture of a pavement is a critical element of the road/tyre interaction and there exist several commonly used descriptions of surface texture. The subject of this part of the investigation is the estimation of aggregate particle (road stone) surface texture, with particular reference to the influence of this texture on the skid resistance of the road pavement and the way it changes as it is polished by traffic in real conditions of pavement exploitation.

Both micro-texture and macro-texture evolve under the effect of traffic and weathering. The most rapid evolution of the friction coefficient occurs in the early stage of using the road pavements after which it stabilizes. In the latter period changes to the friction coefficient are of seasonal nature and depend on the season of the year and on the climate zone. Microscope scale analysis of the surface for test samples supported Mean Texture Depth comprehensive estimation. 


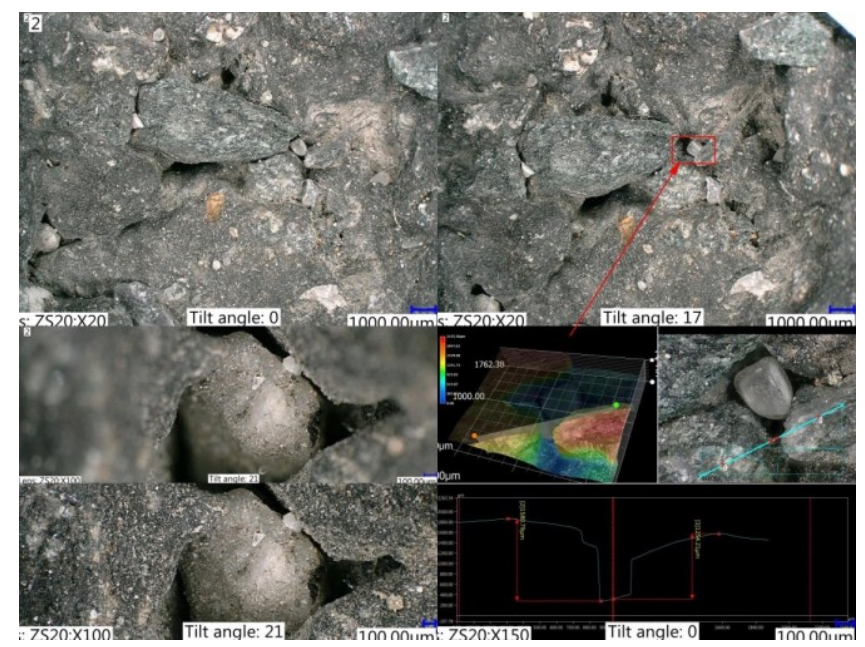

Fig. 4. The surface of standard type of SMA 11 PMB 45/80-45 by microscope investigation.

Microscope scanning methodology, using Keyence VHX-6000, had generated the possibility of $2 \mathrm{D}$ and $3 \mathrm{D}$ analysis of the microtexture of the surface of the whole sample with sample core $\varnothing 200 \mathrm{~mm}$ (Fig. 4), and gave comprehensive observations of material impurity and aggregate polishing. The highest level of polished aggregate was detected for sample No. 3 (DENSIFALT SMA 11) as expected. The main result of the microscope observation of micro-texture of sample 3 was presented in Fig. 5.

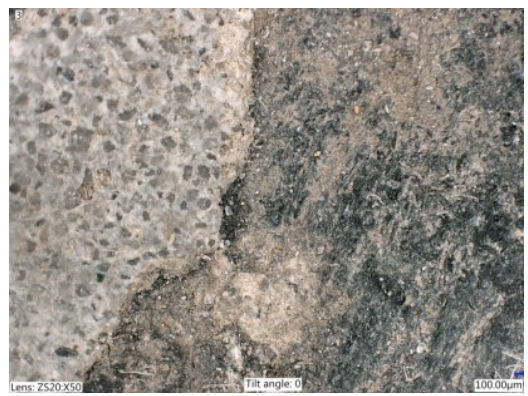

Fig. 5. The sample number 3 with high level of aggregate polishing.

\section{The free space content in the tested samples cut from subsequent pavements}

For the tested surfaces and the corresponding five samples, the free space $\mathrm{V}_{\mathrm{m}} \%$ content was determined. The value of $4.9 \%$ was obtained for sample No. 1, for sample No. 2 the free space content was $1.0 \%$, in the sample No. 3 the content of $6.3 \%$ was the highest in comparison with other test samples. Sample No. 4 is the free content of $2.3 \%$, while sample 5 is $5.3 \%$. Reference documents for the tests of free space in samples taken from the pavement are the following standards: PN-EN 12697-8: 2005, PN-EN 12697-6: 2012, point 10.2 Method B, PN-EN 12697-5: $2010+$ AC: 2012, Method A and PN-EN 13108-20: 2008. According to SST, $\mathrm{V}_{\mathrm{m}}$ should be $\leq 6.0$, while according to WT2-2016 $\mathrm{V}_{\mathrm{m}}$ should be between $2.0 \div 5.0 \%$. Only for two samples No. 1 and No. 4 , the content of free spaces was obtained with values compliant with both SST and WT2-2016. Generally, there is no 
unequivocal glide between MTD test results, for five samples from subsequent pavements, and the results of the free space content determined for these samples.

\section{Conclusions}

The geometry of unevenness of the wearing course of the surface is described by the texture of the surface. Macro-texture is created by unevenness on the pavement surface of the wear layer caused by protruding grains of aggregate. Micro-texture, on the other hand, is created by small irregularities with the depth of tenths of a millimeter caused by the surface roughness of coarse grains and the occurrence of fine grains [3]. MTD research allowed characterizing the macro-texture of five different SMA samples, while microscopic analysis tested the state of the aggregate roughness. The MTD for five samples is $1.1 ; 0.86 ; 0.4 ; 1.4$; $1.4 \mathrm{~mm}$. The largest aggregate polishing occurs in the sample taken from pavement No. 3 , which should be roughened. The applied research methodology associated with the research of macro-texture and micro-texture is the most suitable in the effective assessment of antislip properties of SMA pavement in terms of safety and durability of these surfaces. It would be appropriate and valuable to conduct comparative analysis to assess the depth of macro-texture in the initial period of use of the pavement and in subsequent years of exploitation, as well as to determine the degree of aggregate polishing in the microscopic assessment of test sample cut from the pavements in the subsequent years of road functioning.

\section{References}

1. M. Wasilewska, W. Gardziejczyk, P. Gierasimiuk, Evaluation of Skid Resistance of Exposed Aggregate Concrete Pavement in the Initial Exploitation Period, Roads Bridg. 16 pp. 295-308 (2017). doi:10.7409/rabdim.017.019

2. M.A. Ahammed, S.L. Tighe, Asphalt pavements surface texture and skid resistance exploring the reality, Can. J. Civ. Eng. 39 pp. 1-9 (2012). doi:10.1139/111-109

3. G. Yang, J. Li, J. Zhan, K.C.P. Wang, C. Wang, Wavelet based Macrotexture Analysis for Pavement Friction Prediction, KSCE J. Civ. Eng. 22 pp. 117-124 (2018). doi:10.1007/s12205-017-1165-x

4. M.J. Perry, Road Materials and Pavement Design Role of aggregate petrography in micro-texture retention of greywacke surfacing aggregate Role of aggregate petrography in micro-texture retention of greywacke surfacing aggregate Independent scholar, Road Mater. Pavement Des. 15 pp. 791-803 (2014). doi:10.1080/14680629.2014.923781

5. J. Piłat, P. Radziszewski, Asphalt Pavements (WKŁ, Warsaw, Poland, 2007) 